\title{
A surge in neglected shoulder dislocations and delayed surgical management due to the coronavirus disease 2019 lockdown in India
}

\author{
Dipit Sahu ${ }^{1,2}$, Arun Gupta ${ }^{3}$, Samarjit S. Bansal ${ }^{4}$ \\ ${ }^{1}$ Mumbai Shoulder Institute, Mumbai, India \\ ${ }^{2}$ Department of Orthopaedics, Sir H. N. Reliance Foundation Hospital, Mumbai, India \\ ${ }^{3}$ OJAS Clinic, Agra, India \\ ${ }^{4}$ Aastha Hospital, Mumbai, India
}

Four patients with shoulder problems that were traumatic in etiology presented to us with delays in seeking care ranging from 6 to 12 weeks due to the coronavirus disease 2019 (COVID-19) lockdown. The care of three cases (a 3-month-old neglected anterior shoulder dislocation with a greater tuberosity fracture in a 30-year old man, a 3-month-old neglected anterior shoulder dislocation in a 17-year old boy, and a 2-month-old neglected greater tuberosity fracture in a 31-year old man) was delayed due to the lockdown and the ensuing travel restrictions, while that of one case (a 6-week-old fracture-dislocation of the proximal humerus in a 55-year-old woman) was delayed because the patient was undergoing treatment for COVID-19 at the time of injury. This report intends to present the exceptional circumstances around these cases. The unique treatment challenges and their outcomes are also described to advise the surgeons of the nuances and difficulties in treating these injuries.

Keywords: COVID-19; Shoulder; Neglected disease; Shoulder dislocation; Case report

In February and March 2020, several countries announced a partial or total lockdown in an effort to limit the spread of severe acute respiratory syndrome coronavirus 2, capable of causing coronavirus disease 2019 (COVID-19) [1]. Country-specific guidelines and World Health Organization guidelines specified that non-urgent surgeries should be postponed in view of the prevailing pandemic. To help and guide the scheduling of procedures, orthopedic fracture operations were classified as urgent and surgically necessary procedures [2]. However, for patients with COVID-19, most orthopedic procedures were deferred until the patients subsequently tested negative for infection. During the first 3 months (April-June 2020) of the lockdown in India, there was an overall decrease in the number of orthopedic and trauma surgeries in all hospitals because of the stay-at-home orders and a complete halting of all elective surgeries. Some fractures of the upper limb that could be managed conservatively were preferably treated nonoperatively at our hospital. Elective surgeries, such as rotator cuff repairs, and instability surgeries

Received: April 22, 2021 Revised: September 15, 2021 Accepted: September 26, 2021

Correspondence to: Dipit Sahu

Mumbai Shoulder Institute, Galleria, Hiranandani, Mumbai 400076, India

Tel: +91-22-25717084, E-mail: dip.it@me.com, ORCID: https://orcid.org/0000-0003-1888-4994

Financial support: None.

Conflict of interest: None.

Copyright@ 2021 Korean Shoulder and Elbow Society.

This is an Open Access article distributed under the terms of the Creative Commons Attribution Non-Commercial License (http://creativecommons.org/licenses/by-nc/4.0/) which permits unrestricted non-commercial use, distribution, and reproduction in any medium, provided the original work is properly cited. 
were deferred to a later date. This was done to mobilize resources for the fight against COVID-19. Although fracture surgeries and shoulder trauma decreased in most parts of the world during this period, we started facing challenging shoulder problems due to the social impact of the COVID-19 lockdown. Four patients with shoulder problems that were traumatic in etiology presented to us with care-seeking delays ranging from 6 to 12 weeks due to the COVID-19 lockdown. The care of three patients aged 17 to 31 years was delayed and neglected due to the lockdown and the ensuing travel restrictions; these cases included an (1) anterior shoulder dislocation with a greater tuberosity (GT) fracture in a 30-year-old man (neglected for 3 months), (2) anterior shoulder dislocation in a 17-year-old boy (neglected for 3 months), and (3) GT fracture in a 31-year-old man (neglected for 2 months). Separately, the fourth case was a fracture-dislocation of the proximal humerus in a 55-year-old woman that was managed after a delay of 6 weeks as the patient was undergoing treatment for COVID-19 at the time of her injury. Neglected shoulder problems are not commonly seen in clinical practice, and all four of these cases presented to us after partial lifting of the lockdown. Moreover, these patients' delay in seeking treatment was due to the effects of the COVID-19 lockdown and, as a result, led to unique treatment challenges. This report aims to highlight the etiology and the sudden surge of neglected problems due to COVID-19 lockdown. The unique treatment challenges and their outcomes are also described to apprise surgeons of the nuances and difficulties in treating these injuries.

\section{CASE REPORT}

No ethics approval was required in accordance with the local laws for case report submission. All study subjects gave their consent for participation. Ten shoulder trauma-related urgent surgeries were performed by us between June 15 and August 15, 2020, after the lockdown was partially lifted. Amongst the 10 surgeries, four (described herein) were considered to be delayed and neglected, and the rest were acute fractures of the proximal humerus.

\section{Case 1}

\section{Neglected (3 months) anterior dislocation of the shoulder with a GT fracture}

A 30-year-old man sustained an anterior dislocation of the right shoulder along with a GT fracture (Fig. 1) in the last week of April 2020 after being hit on the shoulder by a stick in a local street fight in a town located 150 kilometers from the main city. This region was served by general primary physicians in primary health services who are only equipped to treat common diseases; as a result, the local doctors provided the patient with a sling, no $\mathrm{X}$-ray imaging or further management for the dislocation was performed. The patient was unable to travel because of the lockdown restrictions and sought our opinion only after a delay of 3 months with symptoms of severe pain with a visual analog scale (VAS) score of 8 points (out of a maximum of 10 points) and restricted use of the affected limb. His functional ability to use the injured limb was limited because he had severe pain and a restricted shoulder range of motion of $-10^{\circ}$ of external rotation, $\mathrm{L} 4$ vertebral level of internal rotation, and $50^{\circ}$ of forward flexion. Computed tomography imaging did not reveal any glenoid bone loss. He was operated on after his COVID-19 status was confirmed to be negative under full personal protective equipment precautions as was the protocol for all surgeries. He underwent open reduction through a deltopectoral approach. During surgery, the following difficulties were encountered: (1) reduction of the GT was not possible without debulking the lateral part of the proximal metaphysis of the proximal humerus as ossification and fibrosis restricted the anterior reduction of the GT and (2) the lesser tuberosity (LT) was not visible even with a medial retraction of the conjoint tendon because of severe medial displacement of the proximal humerus. Hence, a coracoid osteotomy was performed that enabled the conjoint tendon to be retracted further medially and allowed the exposure of the LT and the attached subscapularis. The entire subscapularis had to be detached (later repaired) to allow the reduction of the humeral head to the glenoid. We also released the posterior capsule from the glenoid in order to reduce the head on the glenoid. However, the head kept slipping out anteriorly with even the slightest of rotation of the arm and was found to be unstable because of the severe contractures. Ultimately, the chronicity of the neglected dislocation resulted in severe contractures around the head; hence, two K-wires were used to fix the acromion to the humeral head (Fig. 1B) in order to keep the humeral head stable in the glenoid cavity. The GT was dissected from the surrounding fibrosis and fixed to the humerus with the help of suture anchors. The neglected status of the injury led to an increased surgery duration (3.5 hours) because extensive capsular release, entire subscapularis release, proximal metaphysis debulking to reduce the GT, and $\mathrm{K}$-wire fixation to stabilize the head were required. There were also two instances of uncontrolled bleeding that necessitated packing the wound for 15 minutes until the bleeding subsided. The coracoid tip that had been osteotomized was reattached to its base with the help of cerclage sutures through the tip and the remaining coracoid bone. Postoperatively, there was no neu- 
rovascular deficit or any other complication, and both the K-wires were removed at 4 weeks. This case was more complicated than the other case (case no. 2) of neglected shoulder dislocation because the displaced GT fracture resulted in a loss of lateral restraining forces on the humeral head, causing it to displace far too medially. The patient's pain (VAS score) and external rotation improved from 8 points and $-10^{\circ}$ preoperatively to 3 points and $55^{\circ}$ at 6 months of follow-up. Further, the forward flexion increased from $50^{\circ}$ preoperatively to $80^{\circ}$ at 6 months of follow-up, and internal rotation improved from L4 to the T12 vertebral level. The 6-month follow-up X-ray showed persistent subluxation of the humeral head (Fig. 1C and D), and the patient reported mild pain (VAS score 2/10).

\section{Case 2}

\section{Neglected (3 months) anterior shoulder dislocation}

A 17-year-old boy fell from his bed on May 1, 2020, and sustained an anterior shoulder dislocation of the left shoulder (Fig. 2). He went undiagnosed for 2 months because the local doctors did not perform an X-ray and he could not travel to a more equipped center because of the lockdown. He presented to us with pain
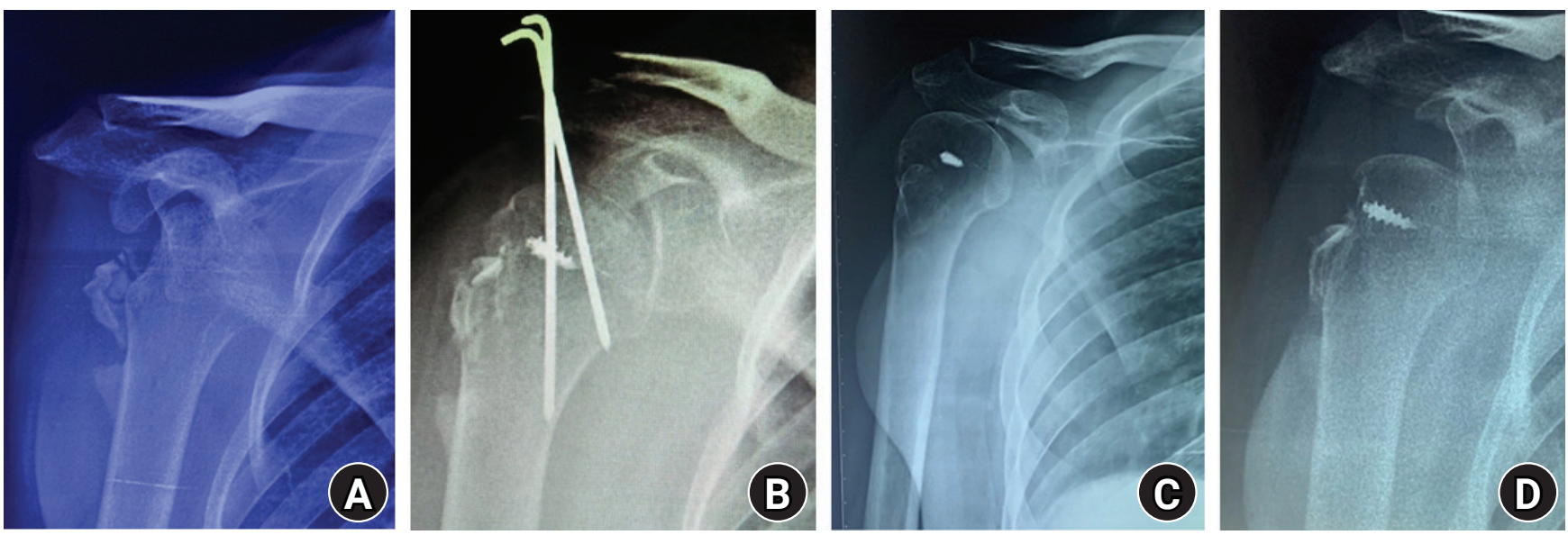

Fig. 1. Case 1. (A) An X-ray showing an anteroposterior view of the right shoulder of a 30-year-old man with a 3-month-old neglected anterior shoulder dislocation along with a greater tuberosity (GT) fracture. (B) A postoperative X-ray showing K-wire fixation from the GT to the humeral head to stabilize the joint. (C) A 6-month follow-up X-ray with arm in external rotation. (D) A 6-month follow-up X-ray with arm in neutral rotation showing subluxation of the humeral head.
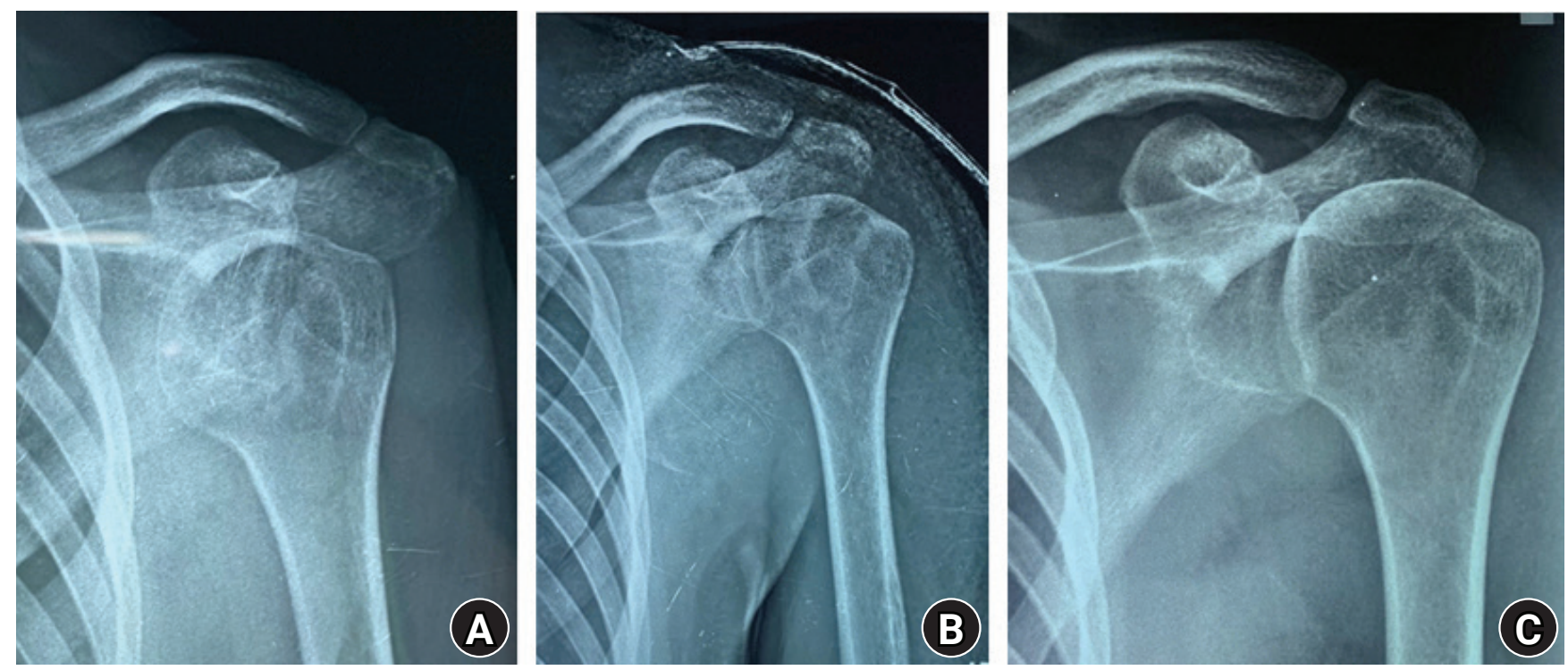

Fig. 2. Case 2. (A) An X-ray showing an anteroposterior view of the left shoulder of a 17-year-old boy with a 3-month-old neglected anterior shoulder dislocation. (B) A postoperative X-ray showing reduced humeral head in the glenoid cavity. (C) A 6-month follow-up X-ray showing a stable glenohumeral joint. 
and limited movement of the shoulder. No closed reduction was attempted because of the chronicity of the problem, and an open reduction approach was planned; he finally underwent open reduction at our facility 3 months after the injury. We used the deltopectoral approach, did not perform a coracoid osteotomy, removed the superior half of the subscapularis, and reduced the head in the glenoid. Intraoperatively, the joint was stable, no $\mathrm{K}$-wire fixation was needed, no capsulolabral repair was attempted, and the subscapularis tendon was repaired. Postoperatively, we did not notice any neurovascular deficit or immediate complications. The surgery duration was 60 minutes, and the duration of the hospital stay was 2 days. The patient's pain (VAS score) and external rotation in adduction improved from 6 points and $0^{\circ}$ preoperatively to 0 points and $60^{\circ}$ at 6 months, while the forward flexion increased from $50^{\circ}$ preoperatively to $140^{\circ}$ and the internal rotation improved from the sacroiliac joint to the T12 vertebral level at 6 months. Additionally, at 6 months of follow-up, the constant score improved to 74 points from a preoperative score of 13 points, and X-ray imaging showed a humeral head concentrically reduced in front of the glenoid.

\section{Case 3}

\section{Neglected (2 months) GT fracture}

A 31-year-old man fell down while riding his two-wheeler on May 10, 2020, and sustained a distal-end radius fracture of the right wrist and GT fracture of the left shoulder (Fig. 3). His wrist fracture was managed by plaster immobilization in a local hospital. The expertise to surgically manage the GT fracture was absent at the local hospital, and no other center's help could be sought due to the limited availability of resources in the area of his residence, the limited functioning of specialized trauma services in the local area, and the travel restrictions in place during the initial lockdown period. After the lockdown was partially lifted, he was referred to us for his GT fracture management (i.e., a delay of 7 weeks from the injury). We fixed his GT in a double-row fashion with two suture anchors medially and a cannulated fully threaded 4-mm screw laterally as a lateral post, using a deltoid-splitting approach. We did not observe any immediate postoperative complications or neurovascular deficit. The fracture could have been managed arthroscopically in the acute period, however as he presented 2 months late, we decided to manage the fracture and the surrounding adhesions using an open deltoid-splitting approach because, in our experience, mobilizing a posteriorly displaced GT fragment in a chronic case is completed more easily via an open approach. The fracture united at 3 months of follow-up and the patient's pain improved from a preoperative VAS score of 5 points to 0 points at 6 months of follow-up; additionally, his elevation, external rotation, internal rotation, and constant score improved from $0^{\circ}, 0^{\circ}$, $\mathrm{L} 1$, and 13 points preoperatively to $110^{\circ}, 50^{\circ}, \mathrm{T} 12$, and 62 points at 6 months of follow-up.

\section{Case 4}

\section{Six-week-old fracture-dislocation of the proximal humerus}

A 55-year-old woman sustained a fracture-dislocation of the left proximal humerus (Fig. 4) after falling from her hospital bed during an episode of epileptic convulsions at a COVID-19 care center on May 3, 2020. The woman was symptomatic with cough and fever, had a past history of epilepsy, and had been admitted to the COVID facility 3 days prior to the incident. However, her
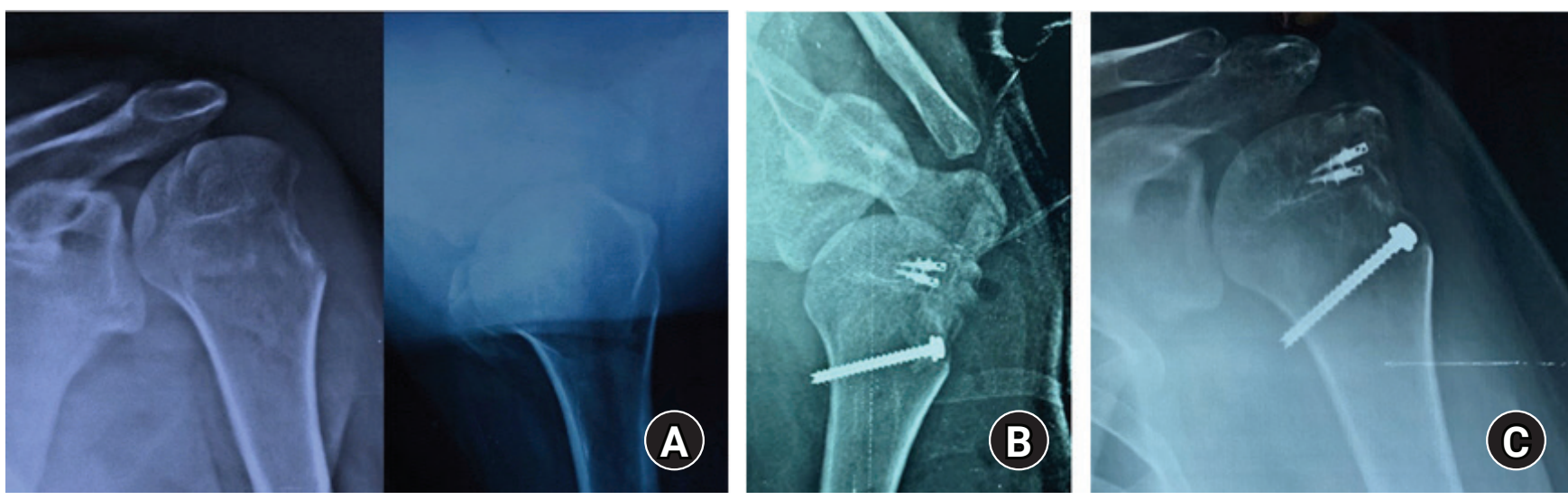

Fig. 3. Case 3. (A) An X-ray showing an anteroposterior view and axial view of the left shoulder in a 31-year-old man with a 2-month-old neglected greater tuberosity (GT) fracture. (B) A postoperative X-ray showing double-row fixation of the GT with the help of two suture anchors as medial row and a 4-mm cannulated screw as a lateral row post. (C) A 6-month follow-up X-ray showing the healed GT fracture. 

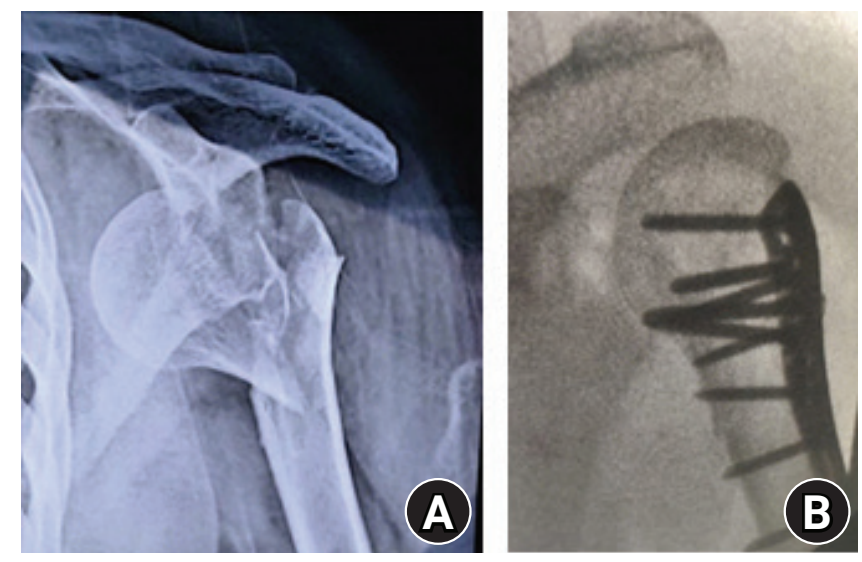

Fig. 4. Case 4. (A) An X-ray showing an anteroposterior view of the left shoulder in a 55-year-old woman with a 6-week-old fracturedislocation of the left proximal humerus. (B) A postoperative X-ray showing precontoured locking plate fixation of the proximal humerus fracture-dislocation.

fracture could not be operated upon at the index hospital because of her concurrent infected status. She was discharged from the COVID-19 facility after 2 weeks; however, confirmation of her fitness for surgery was further delayed due to her uncontrolled epilepsy and COVID-19 status, and she finally underwent operative intervention after a delay of 45 days after swab-testing negative for COVID-19 twice and after her seizures were controlled. She could not afford a humeral head replacement and was not covered by any health insurance policy that would pay for her implant and procedure costs; hence, we fixed her fracture with a precontoured locking plate through a deltopectoral approach. Although there was an increased risk of avascular necrosis, this risk was explained to the patient. The surgery duration was 75 minutes, and the duration of hospital stay was 4 days. This patient was lost to follow-up after 6 weeks.

\section{Comparison with the Pre-COVID-19 Period}

To compare the numbers of neglected trauma cases managed in our unit during the pre-COVID-19 period with the ones managed recently, we retrieved our records of urgent shoulder trauma surgeries performed by our unit in the two 6-month periods preceding the COVID-19 lockdown, i.e., from October 1, 2019, to March 23, 2020, and from April 1 to September 30, 2019. In the former period, we performed 72 urgent shoulder trauma surgeries; of these 72 surgeries, there was one case of a neglected anterior dislocation managed with open reduction and one case of a neglected posterior dislocation managed with open reduction and the McLaughlin procedure. In the latter period, we had performed 65 urgent shoulder trauma surgeries, one of which was a case of neglected anterior dislocation managed by open reduc- tion and one of which was a case of neglected fracture-dislocation of the proximal humerus managed by open reduction and plate fixation. Thus, the rate of neglected injuries corresponds to $3 \%$ of all treated injuries.

\section{DISCUSSION}

The aim of this report was to highlight the recent surge in complicated and neglected shoulder problems due to the social and economic impacts of the COVID-19 lockdown and to discuss the appropriate timing for surgery on a COVID-19-positive patient. Although recent reports indicated that a reduction in the overall incidence of shoulder-related trauma occurred during the COVID-19 lockdown period [3], four neglected presentations within a span of 2 months clearly represents a recent surge in chronic and neglected conditions due to the lockdown because neglected shoulder dislocations are not a commonly treated condition. A perusal of our past records showed that we operated on only two neglected anterior dislocations and one neglected fracture-dislocation within the one year preceding the COVID-19 pandemic. During the COVID-19 lockdown period in 2020, all elective surgeries were suspended at our hospital per the government directive; however, trauma and fracture surgeries were classified as urgent and were allowed to continue. The COVID-19 lockdown not only affected the scientific aspects of fracture care but also impacted its economic and social aspects, and these socioeconomic concerns eventually affected the care of patients. Although neglected shoulder dislocations are unusual, they have been regularly reported in published papers from developing countries [4] as well as developed ones [5]. However, neglected anterior dislocations in the younger age group may have been reported in slightly higher numbers from developing countries. Unless there are extenuating social or medical circumstances, it is unusual for a disabling condition like an anterior shoulder dislocation to be left untreated in younger patients. In our report, amongst the group of three patients whose care was neglected due to lockdown, the maximum age was 31 years and the patient with neglected shoulder dislocation was only 17 years old. Economic factors affecting the rescheduling of orthopedic surgeries have been recently reported from Europe [6], but the social aspects have received scant attention. Even though the socioeconomic impact of COVID-19 lockdown has been felt worldwide, the socioeconomic chiasm is bound to deepen in areas where it already existed. The three patients with neglected trauma were unable to seek proper care during the lockdown period because expert services were lacking and local hospitals were unable to mobilize the necessary resources. Additionally, 
expert orthopedic services to surgically treat the fractures were not available in the patients' area of residence. In India, primary, secondary, and tertiary care centers are established in the order of increasing specialized service availability. Smaller cities and rural areas are served by primary health posts that are only equipped to manage common diseases. For any expert or specialized services, patients are usually referred to community (secondary) health centers or tertiary health centers. Tertiary health centers have the most specialized and advanced facilities but are located primarily in larger urban city areas, and they receive patients referred from primary and secondary centers. Primary health posts lack specialized services. The absence of expert and specialized services near our patients' homes may also have contributed to their conditions being left neglected. Additionally, the travel restrictions in place made it more difficult to seek proper care outside of their area. Two of these patients came from an area 150 kilometers away from the main city, where basic health services are available during normal times but may be limited during the lockdown. We showed that a neglected dislocation could be successfully managed in our 17-year-old patient with good outcomes during short-term follow-up. Meanwhile, the presence of GT fracture along with the dislocated head in the first case presented a challenging situation due to the absence of lateral restraining forces. Notably, this resulted in more severe medial migration of the humeral head; as a result, the surgery was prolonged, and the resulting forward flexion was suboptimal with persistent subluxation of the humeral head. However, the patient's external rotation improved significantly, which enabled him to carry out his daily activities. The prognosis of such severe injuries in a neglected scenario should be determined because they may not always achieve the desired outcome. Arthroplasty options for neglected shoulder dislocation may be available for the older age groups [5], but the options for the younger age group are limited and more nuanced. Open reduction of neglected anterior dislocations has been reported to yield variable results in the literature. Li and Jiang [4] reported high rates of instability after open reduction and coracoid transfer through a subscapularis cut-and-repair technique, but some authors have reported that average to good outcomes are achievable after open reduction with or without acromiohumeral $\mathrm{K}$-wire fixation for stability [7]. Furthermore, conservative neglect has shown poor functional results and, hence, an attempt at open reduction may be made by shoulder surgeons because patients are in pain and have limited functional abilities [7]. A risk of neurovascular complications may also prevent some surgeons from attempting an open reduction in a neglected anterior dislocation, but several reports and our experience show that this fear is unfounded.
According to Siebenbürger et al. [8], delayed head-salvage procedures for fracture-dislocations of the proximal humerus may increase the risk of avascular necrosis of the humeral head. However, a recent report by Trikha et al. [9] stated that good results are achievable even in delayed reconstructions in patients who are younger than 60 years. If a patient with a fracture of the proximal humerus also has a concomitant COVID-19 infection, surgical intervention may have to be deferred in view of the increased risk and dangers of operating on a COVID-19-positive patient. Several authors have recommended that all surgically necessary operations may be performed after two real-time polymerase chain reaction tests are negative and the patient has become asymptomatic. However, some patients may continue to test positive via real-time polymerase chain reaction for up to 3 to 12 weeks after their initial symptoms [10] because viral RNA can be found in the throat in low amounts for up to 12 weeks, even though the symptoms have subsided and the patient has clinically recovered. However, several authors have stated that replication-competent viruses are rarely isolated beyond 10 days from an asymptomatic patient who had earlier tested positive. For a shoulder surgeon, a delay beyond few weeks may be undesirable in view of the entailed difficulties in achieving a proper reduction and consequent compromised results as was seen in our case 1 that had a GT fracture along with a chronic anterior dislocation.

Though recent reports indicate that shoulder trauma decreased in frequency during the COVID-19 lockdown, we are now seeing a surge in neglected shoulder trauma cases, such as neglected anterior dislocation and neglected GT fractures, even though the lockdown was only partially lifted. As the COVID-19 pandemic continues, we may face increased challenges in managing neglected upper limb trauma, either because of the lockdown hardships or concomitant COVID-19 diagnosis. Cases of anterior dislocation that are neglected for a few months can be managed with open techniques with good results; however, treating a concomitant GT fracture along with neglected anterior dislocation may present additional challenges during open reduction, such as extensive adhesions, instability, and prolonged surgical duration and may culminate in suboptimal results.

\section{ORCID}

Dipit Sahu

Samarjit S. Bansal

https://orcid.org/0000-0003-1888-4994

https://orcid.org/0000-0002-0075-0210

\section{REFERENCES}

1. Sahu D, Agrawal T, Rathod V, Bagaria V. Impact of COVID 19 
lockdown on orthopaedic surgeons in India: a survey. J Clin Orthop Trauma 2020;11(Suppl 3):S283-90.

2. DePhillipo NN, Larson CM, O'Neill OR, LaPrade RF. Guidelines for ambulatory surgery centers for the care of surgically necessary/time-sensitive orthopaedic cases during the COVID-19 pandemic. J Bone Joint Surg Am 2020;102:933-6.

3. Gumina S, Proietti R, Polizzotti G, Carbone S, Candela V. The impact of COVID-19 on shoulder and elbow trauma: an Italian survey. J Shoulder Elbow Surg 2020;29:1737-42.

4. Li Y, Jiang C. The effectiveness of the latarjet procedure in patients with chronic locked anterior shoulder dislocation: a retrospective study. J Bone Joint Surg Am 2016;98:813-23.

5. Statz JM, Schoch BS, Sanchez-Sotelo J, Sperling JW, Cofield RH. Shoulder arthroplasty for locked anterior shoulder dislocation: a role for the reversed design. Int Orthop 2017;41:1227-34.

6. Hernigou J, Valcarenghi J, Safar A, et al. Post-COVID-19 return to elective orthopaedic surgery-is rescheduling just a reboot process? Which timing for tests? Is chest CT scan still useful? Safety of the first hundred elective cases? How to explain the "new normality health organization" to patients. Int Orthop 2020;44:1905-13.

7. Sahu D, Rathod V, Phadnis A, Shyam A. Results and complications of head-preserving techniques in chronic neglected shoulder dislocation: a systematic review. J Shoulder Elbow Surg 2021;30:685-94.

8. Siebenbürger G, Van Delden D, Helfen T, Haasters F, Böcker W, Ockert B. Timing of surgery for open reduction and internal fixation of displaced proximal humeral fractures. Injury 2015;46 Suppl 4:S58-62.

9. Trikha V, Singh V, Choudhury B, Das S. Retrospective analysis of proximal humeral fracture-dislocations managed with locked plates. J Shoulder Elbow Surg 2017;26:e293-9.

10. He X, Lau EH, Wu P, et al. Temporal dynamics in viral shedding and transmissibility of COVID-19. Nat Med 2020;26:672-5. 